\title{
Getting Ready to Translate: Cytoplasmic Maturation of Eukaryotic Ribosomes
}

\author{
Vikram Govind Panse*
}

\begin{abstract}
The ribosome is the 'universal ribozyme' that is responsible for the final step of decoding genetic information into proteins. While the function of the ribosome is being elucidated at the atomic level, in comparison, little is known regarding its assembly in vivo and intracellular transport. In contrast to prokaryotic ribosomes, the construction of eukaryotic ribosomes, which begins in the nucleolus, requires $>200$ evolutionary conserved non-ribosomal trans-acting factors, which transiently associate with pre-ribosomal subunits at distinct assembly stages and perform specific maturation steps. Notably, pre-ribosomal subunits are transported to the cytoplasm in a functionally inactive state where they undergo maturation prior to entering translation. In this review, I will summarize our current knowledge of the eukaryotic ribosome assembly pathway with emphasis on cytoplasmic maturation events that render pre-ribosomal subunits translation competent.
\end{abstract}

Keywords: Cytoplasmic maturation of ribosomes - Nuclear transport · Ribosome biogenesis . Ribosome export

The ribosome performs the important task of decoding genetic information to proteins. This universal macromolecular machine is built of two subunits comprising $65 \%$ ribosomal RNA (rRNA) and 35\% ribosomal proteins (r-proteins). In prokaryotes, the large (50S) subunit contains two rRNAs (23S and 5S) and 34 r-proteins, and the small (30S) subunit contains one rRNA (16S) and 21 r-proteins. Eukaryotic ribosomes are larger and more complex than their prokaryotic counterpart. The large (60S) subunit contains three rRNAs (25S, $5.8 \mathrm{~S}, 5 \mathrm{~S})$ and $46 \mathrm{r}$-proteins whereas the small (40S) subunit contains a single rRNA (18S) and 33 r-proteins. Nevertheless, both prokaryotic and eukaryotic ribosomal subunits share a common morphology and core structure. ${ }^{[1]}$ The extra rRNA and rproteins in eukaryotic ribosomal subunits are largely restricted to insertions that loop out of the core structure. While the struc-

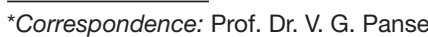
Institute of Biochemistry (IBC) ETH Zürich Schafmattstrasse 18 $\mathrm{CH}-8093$ Zürich

Tel.: +414463362 60

Fax: +414463314 49

E-mail: vikram.panse@bc.biol.ethz.ch
}

ture and function of the mature ribosome is better characterized at the molecular level, ${ }^{[2,3]}$ our knowledge regarding their assembly pathways is still very rudimentary.

\section{Ribosome Biogenesis - 'State of the Art'}

Prokaryotic 50S and 30S subunits can be assembled in vitro from purified rRNA and r-proteins, but this requires conditions that are non-physiological. In vivo rRNA processing and modifying enzymes, and a small number of factors such as RNA helicases, DnaK/Hsp70 and rRNA chaperones systems, GTPases are required for the assembly process (Fig. 1A). These additional factors are dispensable under optimal conditions, but their absence often leads to impaired ribosome synthesis under restrictive conditions. ${ }^{[4]}$ Elegant biophysical analysis of prokaryotic assembly suggests that ribosomal intermediates seen in vitro are not identical to those found in vivo. ${ }^{[5]}$ In contrast, eukaryotic ribosomal subunits cannot be reconstituted in vitro. Building eukaryotic ribosomal subunits in vivo is a complex task and requires concerted action of all three-transcriptional machineries (RNA polymerases I, II and III). The splicing and cellular transport machinery is required to ensure high efficiency and accuracy of ribosome production. In addition, eukaryotic ribosome biogenesis requires $>200$ essential non-ribosomal trans-acting factors that dynamically interact with maturing preribosomal subunits (Fig. 1B). The final outcome of such a spatial and temporal coordination is that, in a growing yeast cell, every second up to 40 nascent ribosomes travel from the nucleolus, the primary site of assembly, to the cytoplasm.

Pioneering work performed in the early 1970 s, by the Planta and Warner laboratories identified the earliest eukaryotic pre-ribosomal particle termed $90 \mathrm{~S}$, that is subsequently processed to yield smaller $66 \mathrm{~S}$ and $43 \mathrm{~S}$ particles, the precursors of $60 \mathrm{~S}$ and 40S subunits, respectively. Today we know that these particles contain the pre-rRNAs, ribosomal proteins and several unknown trans-acting factors that are removed as the pre-ribosomes migrate from the nucleolus to the cytoplasm. In the 1990 s, the application of various genetic screens to the eukaryotic model organism budding yeast has permitted the identification of $\sim 30$ trans-acting factors. ${ }^{[6]}$ Much of this analysis carried out in these yeast mutant strains at the rRNA level have led to a better understanding of the highly ordered steps and positioning of the cleavage sites within pre-rRNA intermediates along the pre-RNA processing pathway. ${ }^{[7]}$ However, the identities of nucleases required for the several rRNA processing steps (Fig. 2) still remain to be elucidated.

Pre-ribosomal subunits need to be transported out of the nucleus to the cytoplasm. In the late 1990s, visual screening approaches in budding yeast were developed to identify factors involved in the nuclear export and the intracellular transport of ribosomal subunits. The in vivo transport assays employed both large-subunit (L25GFP and L11-GFP) and small-subunit reporters (S2-GFP). These transport assays revealed that nucleoporins, the RAN cycle and the general export factor Xpo1/Crm1 are required for nuclear export of $60 \mathrm{~S}$ and $40 \mathrm{~S}$ subunits. ${ }^{[8-10]}$ Screening of temper- 


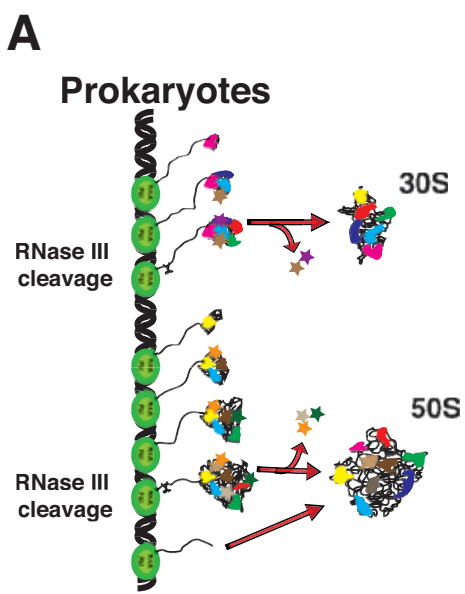

B

\section{Eukaryotes}

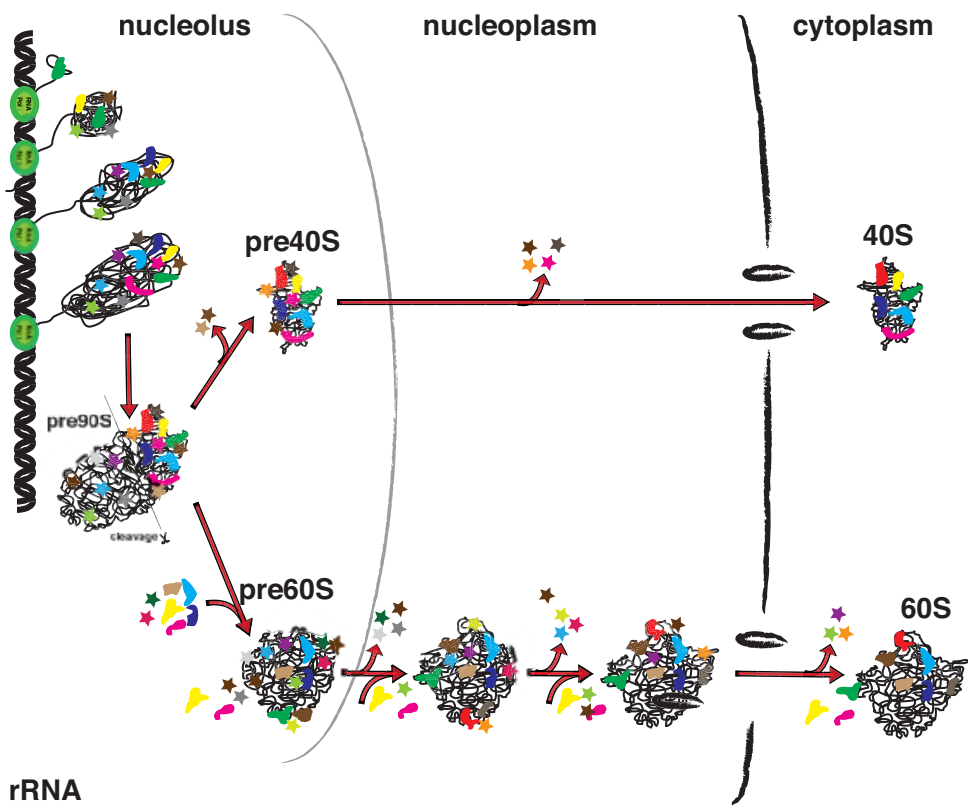

a ribosomal proteins

* trans-acting and transport factors

Fig. 1. Comparison of ribosome biogenesis in prokaryotes and eukaryotes. (A) In prokaryotes, the emerging rRNAs undergo co-transcriptional folding, maturation and assembly with the $r$ proteins. The precursors to the $16 \mathrm{~S}$ and $23 \mathrm{~S}$ rRNAs are cleaved from the nascent transcript by RNase III. Subsequent maturation steps with the help of few non-essential maturation factors lead to the production of mature subunits. (B) The earliest $90 \mathrm{~S}$ precursor derived from RNA polymerase driven transcription is composed of $35 \mathrm{~S}$ pre-rRNA, snoRNPs and 40S biogenesis factors. Following A2 cleavage, the $90 \mathrm{~S}$ splits into pre40S and pre60S subunits. The majority of pre60S factors associate after A2 cleavage. In contrast to pre60S subunits, pre40S subunits undergo few compositional changes as they travel from the nucleolus to the cytoplasm. At the nuclear periphery pre40S and pre60S bind export factors that promote their passage through the nuclear pores. The final maturation steps render pre-ribosomal subunits export competent.

ature-sensitive mutant libraries based on the accumulation of the ribosomal reporters in the nucleolus/nucleoplasm relative to the cytoplasm, revealed several mutants defective in ribosome biogenesis. ${ }^{[11]}$ Despite these fundamental advances the composition of pre-ribosomal particles remained largely unknown until this past decade, when the advent of tandem affinity purification (TAP) protocols in budding yeast combined with sensitive mass spectrometry allowed the isolation and compositional analysis of maturing pre60S and pre40S particles. Further, these analyses have aided the sequential ordering of evolving pre-ribosomal particles along the $60 \mathrm{~S}$ and $40 \mathrm{~S}$ pathways and have provided initial 'biochemical snapshots' of the highly complex and dynamic assembly process. ${ }^{[12-14]}$ These approaches have significantly advanced the field by expanding the inventory of factors that are involved in ribosome biogenesis/export. Nevertheless, the precise role(s) of these factors and their site(s) of action on maturing pre-ribosomal particles are only beginning to be elucidated.

Our current model regarding eukaryotic ribosome assembly and transport has been mainly shaped by genetic, visual screening and proteomic approaches applied to the model organism yeast.[11] Today we know that the emerging precursor 35S rRNA generated by RNA Pol-I driven transcription of rDNA repeats in the nucleolus is co-transcriptionally methylated, pseudouridylated and loaded with a subset of small subunit r-proteins and trans-acting factors, to form the 90S particle (Fig. 1B). Strikingly, the 90S does not contain large subunit r-proteins or trans-acting factors involved in the $60 \mathrm{~S}$ biogenesis pathway. Cleavage at the A2 site releases the pre40S particle and permits the remaining pre-rRNA to assemble with large subunit r-proteins and pre60 biogenesis/maturation factors to form pre60S particles. After separation of the earliest 90S intermediate into pre40S and pre60S particles, the two precursors follow independent biogenesis pathways. Pre40S particles undergo few compositional changes as they travel through the nucleoplasm and are rapidly exported to the cytoplasm. ${ }^{[13]}$ In contrast, pre60S particles associate with $\sim 100$ transacting factors along their biogenesis pathway and therefore undergo dynamic compositional changes as they travel through the nucleoplasm towards the nuclear pore complex (NPC).[12] At distinct stages of biogenesis trans-acting factors are released from pre-ribosomal particles and recycled back to participate in new rounds of biogenesis steps. These events are most likely triggered by several energy-consuming enzymes (ATP-dependent RNA helicases, AAA-ATPases, ABC-ATPases, GTPases) that associate with maturing pre-ribosomal particles. ${ }^{[15,16]}$ These enzymes are believed to provide the energy required to confer directionality to the assembly and maturation process. The site(s) of action of these energy-consuming enzymes on maturing preribosomal particles are only beginning to be uncovered. The stripping/re-modelling action of these diverse energy-consuming enzymes is thought to result in sequential reduction of complexity and acquisition of export competence. The phenomenon of ribosome biogenesis can be compared to an active car assembly line in which several factors aid the formation of specific parts of the ribosome at specific time points.

Export-competent pre-ribosomal particles are exported to the cytoplasm separately upon interaction with the general nuclear export factor Crm1 that directly recognizes a nuclear export sequence (NES). Nmd3 is the only known adaptor 


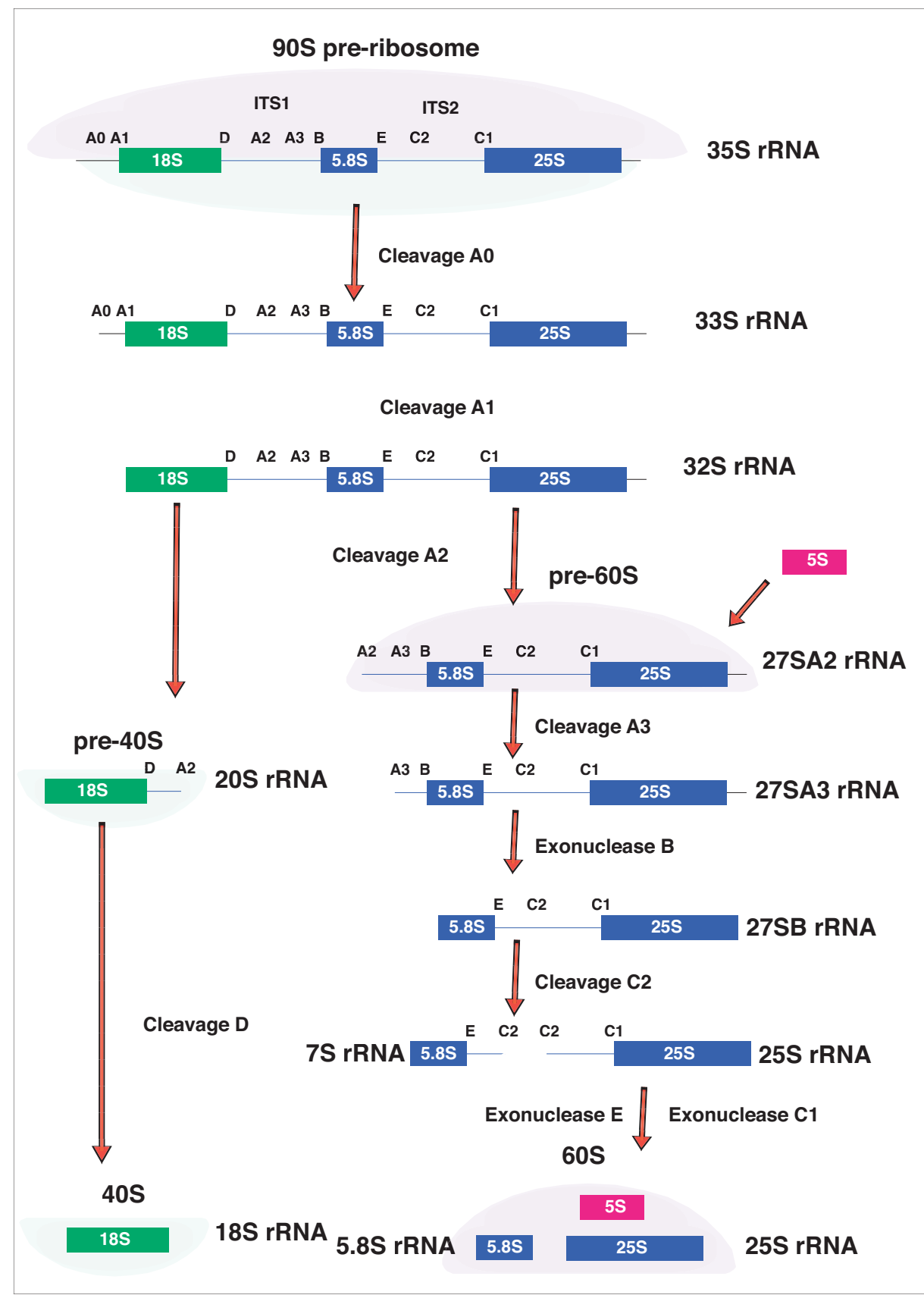

Fig. 2. rRNA processing of eukaryotic ribosomes. The $35 \mathrm{~S}$ pre-rRNA contains sequences for mature 18S, 5.8S, and 25S rRNAs along with additional internal and external spacer sequences. The $35 S$ pre-rRNA transcribed by RNA polymerase I is rapidly modified and processed to produce the $33 S$ pre-rRNA. Cleavage of $33 S$ pre-rRNA at site $A_{0}$ generates the $32 S$ pre-rRNA. The $20 \mathrm{~S}$ and $27 \mathrm{SA}_{2}$ pre-rRNA processing intermediates are generated through internal cleavage of $32 \mathrm{~S}$ prerRNA at the $A_{2}$ site. Subsequent processing and cleavage of $20 S$ and $27 S A_{2}$ pre-rRNAs result in the production of the mature 18S, 25S, and 5.8S rRNAs, respectively. The 5S rRNA is transcribed separately by RNA polymerase III.

between the pre60S particle and Crm1. Additionally, pre60S particles employ multiple trans-acting factors that shield the highly negative charge of the rRNA and position the subunit for entry into the disordered FG-repeats of the transport channel of the NPCs. These factors include the general mRNA export factor Mex67-Mtr2, the shuttling trans-acting factors Arx 1 and Rrp12. Like the pre60S particles, export of the small subunit particles also depends on a functional Crm1 pathway in budding yeast. At least three NES-containing trans-

\section{Cytoplasmic Maturation of Pre-ribosomal Subunits}

The majority of trans-acting factors that associate with both pre60S and pre40S particles during early biogenesis are released and recycled back to the nucleolus prior to nuclear export. However, a few factors remain associated with the particles as they enter the cytoplasm. The release and recycling of these factors, along with the assembly of the few remaining r-proteins, constitute 'late cytoplasmic maturation steps' in the ribosome biogenesis pathway. These steps are crucial not only for completing ribosomal subunit maturation, but also because a failure to recycle a factor to the nucleus leads to its depletion from its nucleolar/nuclear sites of action, inducing pre-rRNA processing delays, assembly defects and impaired nuclear export. ${ }^{[19]}$ What these steps are and understanding why eukaryotic cells have evolved these regulatory steps is a topic that is under intense investigation in my laboratory.

\section{Late Maturation of Pre60S Subunits}

As pre60S particles travel through the nucleoplasm toward the nuclear pore complex, trans-acting factors are released at distinct stages and recycled back to participate in new rounds of biogenesis steps. ${ }^{[15,17,19]}$ the majority of trans-acting factors that associate with pre60S particles during early biogenesis steps are released in the nucleolus/nucleus. However, a few factors (Nmd3, Rlp24, Tif6, Nog1, Arx1, and Alb1) remain associated with pre60S particles and are escorted to the cytoplasm (Fig. 3A). All late pre60S maturation steps investigated to date require cytoplasmic energy consuming GTPases (Kre35, Efl1) and ATPases (Drg1, Hsp70) and cofactors (Sdo1, Rei1, Jjj1) that transiently associate with late pre60S particles.

Upon arrival in the cytoplasm, a series of sequential late maturation events of pre60S subunits are unleashed by the action of the essential AAA-ATPase, Drg1. Drg1 mutants accumulate the nucleolar/nuclearenriched trans-acting factors Rlp24, Nog1, Arx 1 and Tif6 in the cytoplasm where they remain bound to pre60S subunits. AAAATPases typically have discrete substrates, thus it is unlikely that Drg1 acts directly to release each of these proteins. Drg1 appears to target Rlp24 and/or Nog1, with the effect on Arx1 and Tif6 being secondary. Thus an essential mechano-chemical activity of Drg1 initiates cytoplasmic maturation of pre60S subunits.

Rlp24 is closely related to the r-protein Rpl24. Their sequence similarity and apparent mutually exclusive binding to the $60 \mathrm{~S}$ subunit suggest that they bind se- 


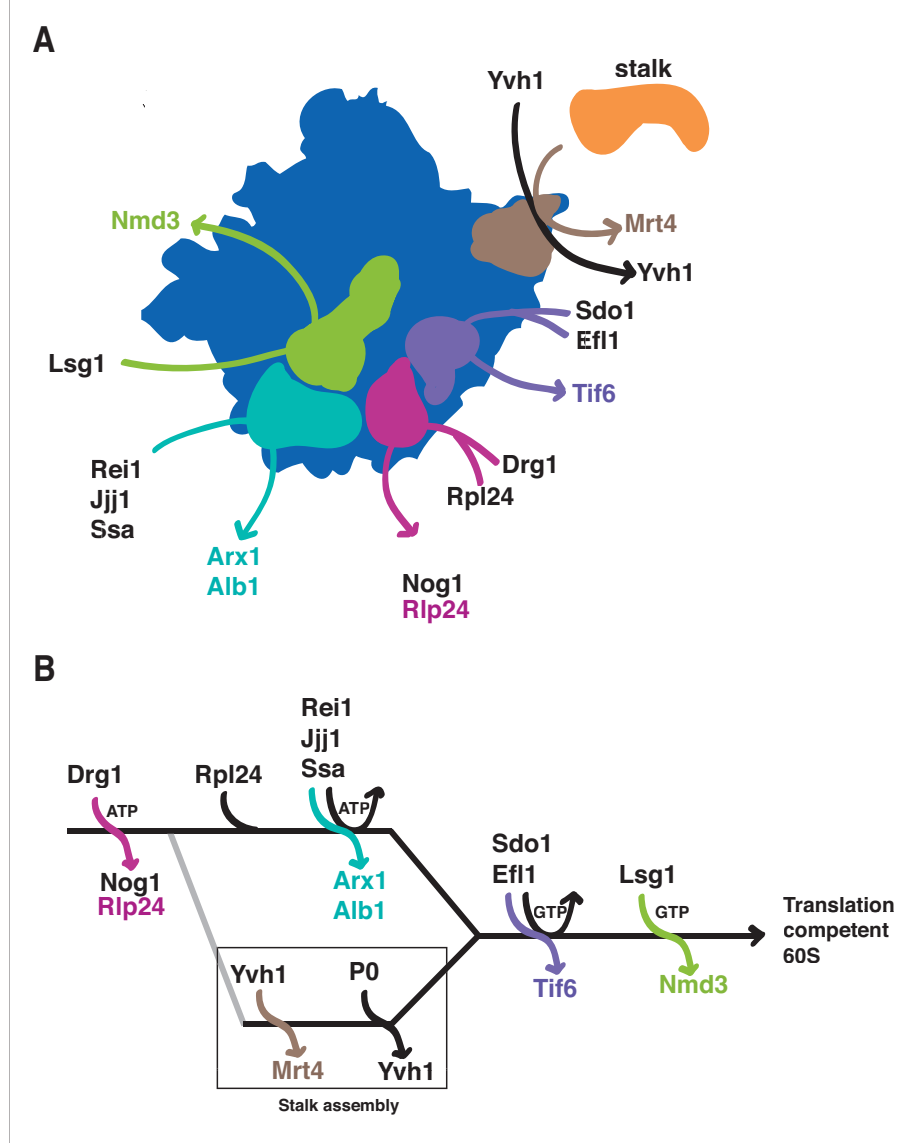

Fig. 3. Cytoplasmic maturation of pre60S subunits. (A) Cartoon summarizing maturation events that occur on pre60S subunits upon their arrival in the cytoplasm. (B) Pathway of 605 maturation in the cytoplasm. Drg1 facilitates the replacement of RIp24 by Rpl24, which then recruits Rei1. The latter, together with Jjj1 and Ssa1/Ssa2, enables the release of the export receptor Arx1, located near the polypeptide exit tunnel. In parallel, Yvh1 enables replacement of Mrt4 with P0 to construct the ribosome stalk. In turn, the stalk recruits the GTPase Efl1 to the GTPaseassociated center to release Tif6 from the subunit joining face of the particle. The release of Tif6 leads to activation of Lsg1 to release export adaptor $\mathrm{Nmd3}$, also from the joining face.

quentially to the same site. Consequently, the release of Rlp24 would be necessary to allow Rpl24 to assemble into the subunit. The incorporation of Rpl24 triggers recruitment of the zinc-finger protein Rei1, therefore establishing an order action of Rei1 after Drg1. Rei1 is not essential but is required for the recycling of Arx 1 , its interacting partner Alb1 and, to a lesser extent, Tif6 to the nucleus. Rei1 works in conjunction with the J protein Jjj1 and the Hsp70 Ssa, an ATPase. reil and jjjl mutants accumulate Arx 1 and Alb1 in the cytoplasm, where they remain bound to pre60S subunits. Deletion of Arx1 suppresses the growth defect of a reil mutant, suggesting that Arx 1 is the direct target of Rei1. Arx1 has evolved from methionyl amino peptidases (MetAPs). Based on similarity of Arx 1 to MetAPs, one would predict that they bind to the same site on the ribosome and that Arx1 would prevent the binding of MetAP. Genetic evidence suggests that Arx 1 binds in the vicinity of the ribosomal protein L25 at the polypeptide exit tunnel. This is an important functional site on the ribosome as L25 interacts with signal recognition particle as well as the translocon in the endoplasmic reticulum.

Cytoplasmic pre60S particles also contain Tif6, a protein that prevents the $60 \mathrm{~S}$ and 40S subunit joining. The GTPase Efl1 and the Swachman-Bodian Syndrome protein ortholog Sdo1 are required to release Tif6. Mutations in either of these factors result in retention of Tif6 on nascent subunits and redistribution of Tif6 to the cytoplasm. Mutations in Tif6 that weaken its affinity for the subunit suppress the growth defects of efll and sdol mutants, providing strong genetic evidence that Tif6 is the primary substrate of Efl1 and Sdol. Efl1 bears strong similarity in sequence to translation elongation factor 2 that facilitates translocation of the ribosome following peptidyl transferase.

The essential nuclear export adapter for pre60S subunits, Nmd3 must also be recycled back to the nucleus. Two proteins, Rpl10 and the GTPase Lsg1, have been implicated in the release of Nmd3. Depletion of Rpl10 or mutations in Rpl10 prevents nuclear recycling of Nmd3. Similarly, mutations in Lsg1 that are predicted to disrupt its GTPase activity also block Nmd3 recycling. These results suggest that Lsg1 induces a conformational change upon Rpl10 loading that stabilizes Rpl10 in the subunit and releases Nmd3.

Recently, my laboratory has identified a new cytoplasmic maturation event that is required for the assembly of the ribosome stalk (Fig. 3A). ${ }^{[20]}$ This structure is essential for recruitment and activation of translation factors, in particular the elongation factors. In yeast, the stalk is composed of $\mathrm{P} 0$ and two heterodimers of $\mathrm{P} 1$ and $\mathrm{P} 2$. $\mathrm{P} 0$ anchors the stalk to the ribosome by binding to the rRNA of Helices 43 and 44 . However, ribosomes are first assembled in the nucleus with Mrt4 in place of P0. Mrt4 is a nuclear paralog of P0, but lacks the domains that recruit translation factors, necessitating an additional step in the maturation pathway: exchange of $\mathrm{P0}$ for Mrt4. We have shown that the dual specificity phosphatase Yvh1 is required for the removal of Mrt4. Interestingly, the protein but not its phosphatase activity is required for this exchange. The assembly of the stalk is a crucial step in the acquisition of functionality of the ribosome.

Recent work from the Johnson laboratory has shed light on the order of events that render pre60S subunits translation competent and provided an initial framework of cytoplasmic maturation events ${ }^{[21]}$ (Fig. 3B). Following the release of Rlp24 by Drg1, the loading of Rpl24 onto the subunit recruits Rei1. In conjunction with Jjj1 and Ssa, these proteins then release Arx1, whose persistence on the subunit impedes the release of Tif6. This suggests a linear pathway from Drg1 release of Rlp24 to Efl1 release of Tif6. However, Tif6 is also mislocalized in $y v h l$ mutants in which stalk assembly is blocked. Considering that the function of the stalk in translation is to recruit and activate GTPases, and that Efl1 is closely related to eEF2, the assembly of the stalk might play a similar role in biogenesis, to recruit Efl1 for the release of Tif6. All these analysis have led to the proposal that the cytoplasmic maturation events in the $60 \mathrm{~S}$ biogenesis are coupled and highly ordered (Fig. 3).

\section{Late Maturation of Pre40S Subunits}

Like the large subunit, the small subunit is also accompanied to the cytoplasm by a handful of proteins (Enp1, Tsr1, Ltv1, Dim1, Dim2, Nob1, Rio2, Hrr25 and Prp43) that mediate its export as well 
as subsequent rRNA processing. However, unlike maturation of the pre60S particle, distinct intermediates of pre $40 \mathrm{~S}$ maturation have yet to be isolated. Cytoplasmic maturation of pre $40 \mathrm{~S}$ particles involves two major events: a structural rearrangement to generate the 'beak' structure of the mature 40S subunit, and the final endonucleolytic cleavage of the pre-rRNA to yield mature 18S rRNA. ${ }^{[22,23]}$

The essential kinase Hrr25 phosphorylates the ribosomal protein S3 as well as factors Ltv1 and Enp1. When phosphorylated S3 is weakly associated with the 40S subunit and is found instead in a sub-complex with Ltv1 and Enp1. Subsequent dephosphorylation is required for the stable incorporation of Rps 3 into the small subunit and the corresponding production of the 'beak' within the head domain of the small subunit. This cycle of phosphorylation and dephosphorylation appears to be critical for the stable association of Rps3 with the small subunit and for maturation of the 'beak' domain, and potentially permitting rapid nuclear export of pre40S subunits. However, a cytoplasmic phosphatase responsible to sustain this cycle still remains unknown.

A second universally conserved cytoplasmic event is cleavage of the small subunit in the processing of 20S rRNA to generate the mature $18 \mathrm{~S}$ rRNA. The nuclease responsible for this cleavage appears to be Nob1. Surprisingly, Nob1 is recruited during early nuclear biogenesis steps of the pre $40 \mathrm{~S}$ subunits. Why this cleavage step occurs only upon the arrival of pre40S subunits in the cytoplasm is not understood. Recent work uncovered a negative synergistic interaction between Ltv1 and the RNA helicase Prp43 that can be rescued by overexpression of Nob1,[23] leading to the proposal that Prp43 drives a conformational change in the pre40S particle that allows Nob1 access to its RNA substrate. Cleavage also requires the essential kinase Rio2. Because Rio2 is also required for the recycling of Ltv1, Enp1, Nob1 and Enp1, it is not yet clear if Rio2 promotes cleavage of $20 \mathrm{~S}$ and thereby allows the recycling of associated proteins or if it promotes a conformational change that releases the pre40S factors allowing Nob1 to efficiently access the cleavage site.

\section{Conclusions and Perspectives}

Eukaryotic ribosomes are assembled in the nucleus in an environment that is physically separated from translation. The general picture that is emerging from studies of pre-ribosome maturation is that in addition to this physical barrier, assembling subunits are also packaged in a functionally inactive state, lacking critical r-proteins that provide functionality to the ribosome and containing trans-acting factors that prevent their function. Thus, in addition to physical compartmentalization, there is functional compartmentalization of preribosomal subunits.

Why export functionally inactive subunits? One possibility is that ribosomes are held in a functionally inactive state to facilitate their transport to the cytoplasm. Analogous to mRNAs that are translationally repressed during transport, a similar phenomenon may occur with ribosomes. This could provide a means of targeting newly made ribosomes to specific cites in a cell, and avoiding engaging with mRNAs and translation factors along the way. Ribosomal subunits may be transported to a region of cell growth such as growing bud in yeast, or a growth cone in a neuron or to a site of active translation such as the ER in a cell devoted to secretion.

Why is there a requirement for a specialized set of release factors in the cytoplasm for each shuttling trans-acting factor? Perhaps each of these factors provides a mechanism for quality control of ribosome synthesis. Nuclear assembly of ribosomes might require "structural proofreading' to efficiently recruit biogenesis factors and transport factors. However, in the cytoplasm, where the ribosomal subunits engage with mRNAs, there may be an opportunity for 'functional proof-reading' to ensure that only active ribosomes are released for translation. The release factor Efl1 closely resembles elongation factor 2 in sequence and therefore likely interacts with the subunit in a manner similar to eEF2. After assembly of the stalk, Efl1 recruitment could 'test' the GTPase activating center of the ribosome, releasing Tif6 in the process. The utilization of a translation-like factor in biogenesis might therefore sense translation competence of the $60 \mathrm{~S}$ subunit prior to engaging with the pre-initiation complex (40S bound to mRNAs).

Given the importance for correctly translating the genetic code, eukaryotic cells are likely to possess quality control mechanisms to monitor ribosome biogenesis. Cytoplasmic maturation steps in both the $40 \mathrm{~S}$ and $60 \mathrm{~S}$ biogenesis pathways appear to activate subunits by removing 'gatekeeping' factors and adding functionality. These steps could therefore ensure only functional ribosomal subunits enter into translation. How does the cell sense functional ribosomal subunits and how would such a sensing mechanism work? Clearly, a detailed spatio-temporal and molecular understanding of these maturation steps in vivo will shed light on the importance of these evolutionary conserved steps.

\section{Acknowledgements}

I thank M. B. Faza and S. Schütz for preparing the figures. I apologize to all the authors whose primary work has not been cited due to space limitations. V. G. Panse is supported by grants from the Swiss National Science Foundation, the Swiss Federal Institute of Technology and the European Research Council (EURIBIO-260676) and, a recipient of a Swiss National Science Foundation Professorship and

a European Research Council Starting Grant Award.

Received: July 18, 2011

[1] D. J. Taylor, B. Devkota, A. D. Huang, M. Topf, E. Narayanan, A. Sali, S. C. Harvey, J. Frank, Structure 2009, 17, 1591

[2] A. Ben-Shem, L. Jenner, G. Yusupova, M Yusupov, Science 2010, 330, 1203.

[3] J. Rabl, M. Leibundgut, S. F. Ataide, A. Haag, N. Ban, Science 2011, 331, 730.

[4] A. E. Hage, D. Tollervey, RNA Biol. 2004, 1, 10

[5] Z. Shajani, M. T. Sykes, J. R. Williamson, Annu. Rev. Biochem. 2011, 80, 501 .

[6] D. Kressler, P. Linder, J. de La Cruz, Mol. Cell Biol. 1999, 19, 7897.

[7] J. Venema, D. Tollervey, Аnnu. Rev. Genet. 1999, 33, 261.

[8] E. Hurt, S. Hannus, B. Schmelzl, D. Lau, D. Tollervey, G. Simos, J. Cell Biol. 1999, 144 389.

[9] T. Stage-Zimmermann, U. Schmidt, P. A. Silver, Mol. Biol. Cell 2000, 11, 3777.

[10] P. Milkereit, D. Strauss, H. Kühn, O. Gadal, H Lechner, E. Hurt, H. J. Tschochner, Biol. Chem. 2003, 278, 4072 .

[11] H. Tschochner, E. Hurt, Trends Cell Biol. 2003, $13,255$.

[12] T. A. Nissan, J. Bassler, E. Petfalski, D Tollervey, E. Hurt, EMBO J. 2002, 21, 5539.

[13] P. Grandi, V. Rybin, J. Bassler, E. Petfalski, D. Strauss, M. Marzioch, T. Schäfer, B. Kuster, H. Tschochner, D. Tollervey, A.-C. Gavin, E. Hurt, Mol. Cell 2002, 10, 105.

[14] T. Schafer, D. Strauss, E. Petfalski, D. Tollervey, E. Hurt, EMBO J. 2003, 22, 1370.

[15] D. Kressler, E. Hurt, J. Bassler, Biochim. Biophys. Acta 2010, 1803, 673.

[16] B. S. Strunk, K. Karbstein, RNA 2009, 15, 2083.

[17] I. Zemp, U. Kutay, FEBS Lett. 2007, 581, 2783

[18] A. Kohler, E. Hurt, Nat. Rev. Mol. Cell Biol. 2007, 8, 761.

[19] V. G. Panse, A. W. Johnson, Trends Biochem. Sci. 2010, 35, 260.

[20] S. Kemmler, L. Occhipinti, M. Veisu, V. G Panse, J. Cell Biol. 2009, 186, 863.

[21] K. Y. Lo, Z. Li, C. Brussiere, S. Bresson, E. M. Marcotte, A. W. Johnson, Mol. Cell 2010, 39, 196.

[22] T. Schäfer, B. Maco, E. Petfalski, D. Tollervey, B. Böttcher, U. Aebi, E. Hurt, Nature 2006, 441 , 651.

[23] B. Pertschy, C. Schneider, M. Gnädig, T. Schäfer, D. Tollervey, E. Hurt, J. Biol. Chem. 2009, 284, 35079 . 